\title{
Shock wave loading and spallation of copper bicrystals with asymmetric $\Sigma 3\langle 110\rangle$ tilt grain boundaries
}

\author{
Sheng-Nian Luo, ${ }^{1, a)}$ Timothy C. Germann, ${ }^{1}$ Davis L. Tonks, ${ }^{1}$ and Qi $A n^{1,2}$ \\ ${ }_{1}^{1}$ Los Alamos National Laboratory, Los Alamos, New Mexico 87545, USA \\ ${ }^{2}$ Materials and Process Simulation Center, California Institute of Technology, Pasadena, \\ California 91125, USA
}

(Received 2 August 2010; accepted 29 September 2010; published online 12 November 2010)

\begin{abstract}
We investigate the effect of asymmetric grain boundaries (GBs) on the shock response of $\mathrm{Cu}$ bicrystals with molecular dynamics simulations. We choose a representative $\Sigma 3\langle 110\rangle$ tilt GB type, $(110)_{1} /(114)_{2}$, and a grain size of about $15 \mathrm{~nm}$. The shock loading directions lie on the GB plane and are along [00 $\overline{1}]$ and $[22 \overline{1}]$ for the two constituent crystals. The bicrystal is characterized in terms of local structure, shear strain, displacement, stress and temperature during shock compression, and subsequent release and tension. The shock response of the bicrystal manifests pronounced deviation from planar loading as well as strong stress and strain concentrations, due to GBs and the strong anisotropy in elasticity and plasticity. We explore incipient to full spallation. Voids nucleate either at GBs or on GB-initiated shear planes, and the spall damage also depends on grain orientation.
\end{abstract}

(C) 2010 American Institute of Physics. [doi:10.1063/1.3506707]

\section{INTRODUCTION}

It has long been recognized that shock response (plasticity and spall damage) may depend strongly on microstructure, e.g., the characteristics of grains and grain boundaries (GBs) in anisotropic materials. ${ }^{1-3}$ Shock experiments ${ }^{1}$ have been routinely performed on polycrystalline solids along with very limited molecular dynamics (MD) simulations. ${ }^{4-6}$ Meyers and Carvalho ${ }^{7}$ modeled the shock front irregularities of polycrystalline solids under the hydrodynamic assumption. Given the vast number of GB types and grain characteristics, it is highly desirable to investigate some elemental processes, e.g., shock response of columnar crystals or bicrystals, so we can gain certain specific insights without being overwhelmed by the complexities of abundant random GBs. Recently, Cao et $a l .{ }^{8}$ examined deformation twinning in diffusion-bonded $\mathrm{Cu}[01 \overline{1}] /[100]$ bicrystal recovered from gas-gun shock wave experiments, and Peralta et al. ${ }^{9}$ studied incipient spall damage in $\mathrm{Cu}$ multicrystals (quasicolumnar) recovered from laser-driven flyer plate experiments. We performed MD shock simulations of [100] columnar nanocrystalline $\mathrm{Cu}$ (Ref. 10) (as well as single crystal $\mathrm{Cu}$ along different crystallographic directions ${ }^{11}$ ). The deviation of shock response from perfect planarity due to microstructure has been investigated with spatially resolved diagnostics (normally recording the movement at free surfaces or the targetwindow interfaces), including line-imaging velocimetry and two-dimensional (2D) displacement interferometry. ${ }^{12-14}$ It would be interesting to model such surface movements with MD in order to help interpret these surface measurements.

As a first attempt, here we perform MD shock simulations of $\mathrm{Cu}$ bicrystals with $\Sigma 3\langle 110\rangle$ asymmetric tilt GBs. Our simulations show that the crystal anisotropy and GBs induce strong deviations of shock response from perfect planarity

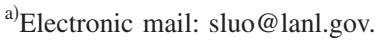

through crosstalk between the two constituent crystals, as well as pronounced stress and strain concentrations. Both plasticity and spall damage are orientation dependent in the bicrystals. In this work, Sec. II presents the methodology for MD simulations and analysis. Results and discussion are addressed in Sec. III, followed by summary/conclusions in Sec. IV.

\section{METHODOLOGY}

The GB characteristics of coincidence site lattice $\Sigma 3\langle 110\rangle$ tilt GBs were investigated previously, including two symmetric and 23 asymmetric tilt GBs with a misorientation angle of $70.53^{\circ}$ about the tilt axis $[1 \overline{1} 0] . .^{15}$ We choose an asymmetric GB type, $(110)_{1} /(114)_{2}$, which has an inclination angle of $35.36^{\circ}$ and an intermediate GB energy of $426.9 \mathrm{~mJ} \mathrm{~m}^{-2}$; the GB normals for grains 1 and 2 (denoted with subscripts) in this bicrystal are [110] and [114], respectively. ${ }^{15}$ We define the tilt axis $[1 \overline{1} 0]$ as the $z$-axis and the GB normals as the $y$-axis, and therefore, the $x$-axis is

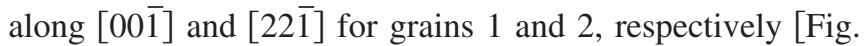
1; visualization uses AtomEye (Ref. 16)]. We first construct a bicrystal with edge lengths of $21.7 \mathrm{~nm} \times 30.6 \mathrm{~nm}$ $\times 15.3 \mathrm{~nm}$ (864 000 atoms). The grain size for each grain (the width along the $y$-axis) is about $15 \mathrm{~nm}$ by construction.

The atomic interactions in $\mathrm{Cu}$ are described with an accurate embedded-atom-method potential. ${ }^{17}$ Our MD simulations use the Institut für Theoretische und Angewandte Physik MD code. ${ }^{18}$ The as-constructed bicrystal is relaxed with the conjugate gradient method, followed by thermalization at the ambient conditions with the constant-pressuretemperature ensemble and three-dimensional (3D) periodic boundary conditions. The resulting bicrystal is taken as the flyer plate in our shock wave simulations (see below). The target configuration is obtained via replicating the flyer plate 


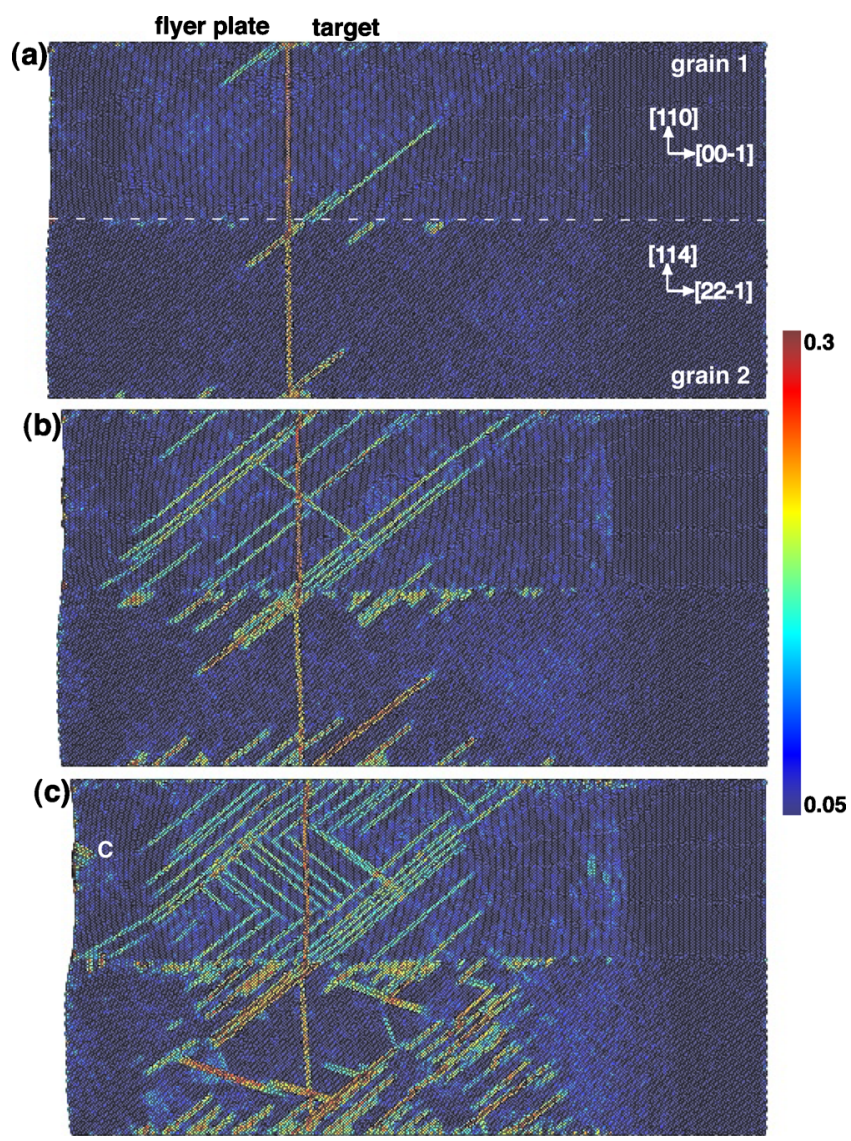

FIG. 1. (Color online) Atomic configurations at $t=6 \mathrm{ps}$ for shock loading with different $u_{\mathrm{p}}$, projected along the tilt axis [1 $\left.1 \overline{1} 0\right]$ (i.e., onto the $x y$-plane). Shock loading direction (the $x$-direction) is along [001] for grain 1 and [221] for grain 2. The dashed line denotes a GB. $u_{\mathrm{p}}=0.375 \mathrm{~km} \mathrm{~s}^{-1}$, $0.5 \mathrm{~km} \mathrm{~s}^{-1}$, and $0.625 \mathrm{~km} \mathrm{~s}^{-1}$ for (a)-(c), respectively. Color coding is based on $\eta^{\mathrm{vM}}$.

configuration by two times along the $x$-axis $(43.4 \mathrm{~nm}$ $\times 30.6 \mathrm{~nm} \times 15.3 \mathrm{~nm}$ or 1728000 atoms), and then equilibrated further at the ambient conditions.

The flyer plate and target configurations are assembled along the $x$-axis (2 592000 atoms). We denote the desired shock state particle velocity (or equivalently, the piston velocity) as $u_{\mathrm{p}}$. The flyer plate and target are assigned initial velocities of $4 / 3 u_{\mathrm{p}}$ and $-2 / 3 u_{\mathrm{p}}$ along the $x$-axis, respectively, before impacting each other. Here we explore $u_{\mathrm{p}}$ $\approx 0.375,0.5$, and $0.625 \mathrm{~km} \mathrm{~s}^{-1}$. Shock simulations use the microcanonical ensemble. ${ }^{11,19,20}$ Periodic boundary conditions are applied along the $y$ - and $z$-axes (not the $x$-axis) to mimic one-dimensional (1D) strain loading. The nonimpact sides of the flyer plate and target are free surfaces. The time step for integrating the equations of motion is $1 \mathrm{fs}$, and the shock run durations are up to 45 ps. Our simulations examine the shock response of repeating bicrystals in the nanolayered structure. (There are a GB located at the center and a GB split between the top and bottom of the simulation cell along the $y$-axis.) The shock direction differs drastically for grains

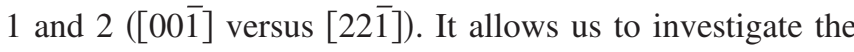
effect of crystal anisotropy on the shock response.

Neglecting the heterogeneities normal to the shock direction (i.e., on the cross-sections), we divide the simulation

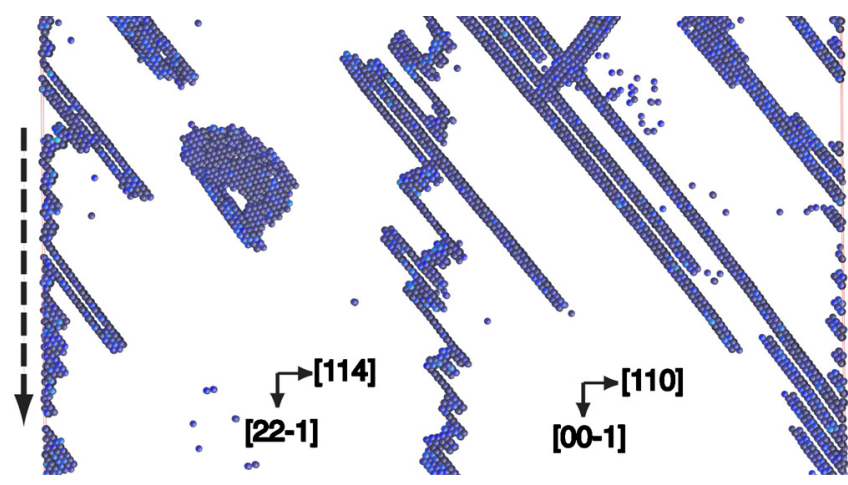

FIG. 2. (Color online) Atom configuration in the shocked region at $t$ $=6 \mathrm{ps}$ for $u_{\mathrm{p}}=0.5 \mathrm{~km} \mathrm{~s}^{-1}$. Centrosymmetry parameter is calculated for each atom, and only non-fcc atoms are shown. Dashed arrow: shock direction. Also see Fig. 1(b).

cell into fine bins only along the $x$-direction (the 1D binning analysis ${ }^{11}$ ), and the average physical properties are obtained within each bin, such as density $(\rho)$, stress $\left(\sigma_{i j}\right)$, particle velocity and temperature $(T)$ profiles along the $x$-axis at different stages of compression, release and tension. $(i, j=1,2$, and 3 , corresponding to $x, y$, and $z$, respectively.) The centerof-mass velocity of a bin is removed when calculating $T$ and $\sigma_{i j} . \sigma_{i j}$ for each bin is the average of virial stresses plus thermal contributions. ${ }^{21}$ We perform uniform (nonshock) compression simulations of a cubic supercell with the microcanonical ensemble and then the binning analysis with different bin widths. We choose the minimum bin width with which the binning analysis matches the direct MD output of the stress tensor for the whole system. A bin width of $5 \AA$ is sufficient for our purpose, and it is $5 \AA$ or more in this work. The free surface velocity $\left(u_{\mathrm{fs}}\right)$ versus time $(t)$ is obtained from the particle velocity evolution on the target free surface. Similarly, 2D and 3D binning analyses (i.e., including binning along the $y$ - or $z$-axis as well) can be performed to better resolve spatially GBs and different grains.

We characterize the local deformation and local structure around an atom with the local von Mises shear strain $\left(\eta^{\mathrm{vM}}\right)$ (Refs. 22 and 23) and centrosymmetry parameter, ${ }^{24}$ both useful for visualizing crystal plasticity. $\eta^{\mathrm{vM}}$ may reveal shear "bands" within a grain and GB sliding. Centrosymmetry parameter can distinguish different atomic packing orders, including the original face-centered-cubic (fcc) packing, hexagonal-close-packed (hcp) stacking faults and twins, and other defects in non-close-packed structure (Fig. 2). Atoms in the slip bands can be in the hcp packing during plastic deformation of $\mathrm{Cu}$, thus a manifestation of crystal plasticity. ${ }^{10,22}$ To characterize the extent of deviation from planar shock, we also calculate the displacement field $D_{i}(x, y, z)$, simply defined for each atom relative to its preshock position.

The shock or Hugoniot state (denoted with a subscript $\mathrm{H})$ parameters are obtained from the 1D profiles. We define $\tau$ as the maximum shear stress, and $2 \tau=\sigma_{x x}-\left(\sigma_{y y}+\sigma_{z z}\right) / 2$. $|2 \tau|(t)$ is obtained in 1D-3D during compression or tension. The tensile strain rate and spall strength $\left(\sigma_{\mathrm{sp}}\right.$, i.e., the 

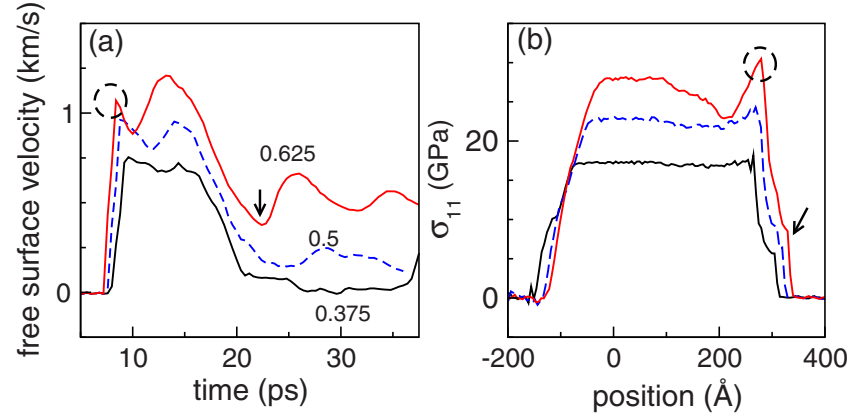

FIG. 3. (Color online) 1D analyses: (a) The target free surface velocity histories, $u_{\mathrm{fs}}(t)$, for different $u_{\mathrm{p}}$ (numbers; in $\mathrm{km} \mathrm{s}^{-1}$ ). (b) The stress profiles $\sigma_{11}(x)$ at $t=6 \mathrm{ps}$ at the corresponding $u_{\mathrm{p}}$.

maximum tensile stress $-\sigma_{x x}$ ) are evaluated in situ right before spallation near the spall zone(s) from 1D (2D or 3D) analysis. $^{11}$

\section{RESULTS AND DISCUSSION}

The impact yields shock waves propagating into the target and the flyer plate, which are then reflected at the respective free surfaces as centered simple rarefaction (release) fans, and their interaction induces an evolving tensile region and spall in the target for sufficiently strong shocks. Structure changes and shear may occur during compression and tension and lead to plastic deformation but can be partially reversed during release. Upon spall, the tensile stress is reduced, inducing (re)compression waves propagating toward the free surfaces. Spall reduces local tension and raises the local temperature. The waves are then trapped and reverberate between the spall "plane" (if developed) and target (or flyer plate) free surface.

1D analysis of shock loading (planar shocks) is of practical use for experiments and modeling. Physical properties are only resolved spatially along the shock or $x$ direction in the 1D analysis; shown in Figs. 3 and 4 are some examples. The shock-release-spall sequence is manifested in the free surface velocity histories [Fig. 3(a)]. Figure 3(b) shows snapshots of in situ stress wave profiles with leading shock front, shock plateau, and trailing release originating from the flyer plate free surface. The tensile stress profiles are obtained at later stages (Fig. 4).

The shock fronts in stress profiles $\left[\sigma_{11}(x)\right.$, Fig. 3(b) $]$ are similar for three different $u_{\mathrm{p}}$, showing a precursor (arrow) and "relaxation" from the peak (circle). These features are more pronounced at higher $u_{\mathrm{p}}$; they are also identified in $u_{\mathrm{fs}}(t)$ but smeared [Fig. 3(a)]. The precursor is normally termed as elastic precursor in 1D analysis, a main indicator of the elastic-plastic transition. However, this precursor is not necessary a result of such transitions; e.g., the compression plasticity is negligible for $u_{\mathrm{p}}=0.375 \mathrm{~km} \mathrm{~s}^{-1}$ (see below). Shock plateaus are well achieved in $\sigma_{11}(x)$ but not in $u_{\mathrm{fs}}(t)$. The differences in wave front features and shock plateau between $u_{\mathrm{fs}}(t)$ and $\sigma_{11}(x)$ are likely due to the complex wave interactions near the free surface. $\sigma_{11, \mathrm{H}}$ is $\sim 17.1 \mathrm{GPa}$, 22.6 GPa, and $27.5 \mathrm{GPa}$, and $T_{\mathrm{H}}, \sim 410 \mathrm{~K}, 460 \mathrm{~K}$, and 550

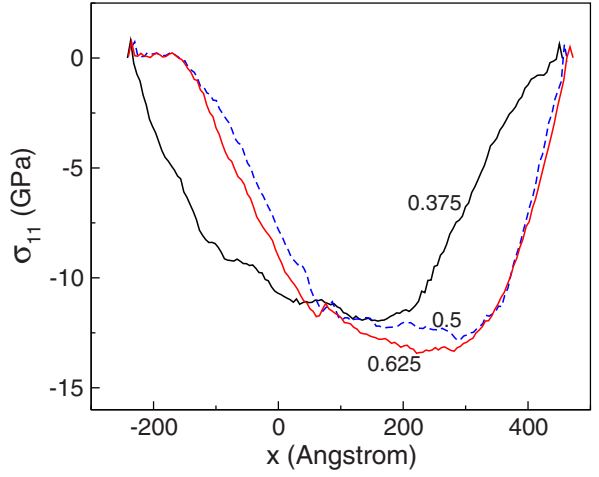

FIG. 4. (Color online) $1 \mathrm{D}$ analysis of the tensile stress profiles $\sigma_{11}(x)$ at $t$ $=22 \mathrm{ps}, 18 \mathrm{ps}$, and $18 \mathrm{ps}$ for $u_{\mathrm{p}}=0.375 \mathrm{~km} \mathrm{~s}^{-1}, 0.5 \mathrm{~km} \mathrm{~s}^{-1}$, and $0.625 \mathrm{~km} \mathrm{~s}^{-1}$, respectively.

$\mathrm{K}$, for $u_{\mathrm{p}}=0.375 \mathrm{~km} \mathrm{~s}^{-1}, 0.5 \mathrm{~km} \mathrm{~s}^{-1}$, and $0.625 \mathrm{~km} \mathrm{~s}^{-1}$, respectively. The corresponding maximum values of $|2 \tau|$ are $\sim 8.1 \mathrm{GPa}, 10.8 \mathrm{GPa}$, and $12.5 \mathrm{GPa}$.

Spall zones can be identified within the target from such profiles as $\rho(x)$, and are characterized by locally reduced density, reduced tensile stress, and elevated temperature. One spall zone is identified from such profiles at $x \approx 200 \AA$ for $u_{\mathrm{p}}=0.5$ and $0.625 \mathrm{~km} \mathrm{~s}^{-1}$, and none for $u_{\mathrm{p}}=0.375 \mathrm{~km} \mathrm{~s}^{-1}$ given the fluctuations in the profiles. ("Incipient" spall does occur for $u_{\mathrm{p}}=0.375 \mathrm{~km} \mathrm{~s}^{-1}$ with a few isolated nanovoids; see below). The recompression following spall is registered in $u_{\mathrm{fs}}(t)$ as a pullback characteristic of spallation. Pullback is only observed for $u_{\mathrm{p}}=0.5$ and $0.625 \mathrm{~km} \mathrm{~s}^{-1}$ [e.g., the arrow in Fig. 3(a)], consistent with in situ results; the spall-related wave reverberations are also evident.

The maximum tensile stress obtained from the 1D stress profiles is $\sim 11.8 \mathrm{GPa}, 12.8 \mathrm{GPa}$, and $13.5 \mathrm{GPa}$ for $u_{\mathrm{p}}$ $=0.375 \mathrm{~km} \mathrm{~s}^{-1}, 0.5 \mathrm{~km} \mathrm{~s}^{-1}$, and $0.625 \mathrm{~km} \mathrm{~s}^{-1}$, respectively. For the latter two cases with spallation, it represents nominally the spall strength $\sigma_{s p}$ (Refs. 11 and 20). We examined systematically $\sigma_{s p}$ of single crystal $\mathrm{Cu}$ along different crystallographic directions. ${ }^{11}$ For example, $\sigma_{s p}$ is about $16 \mathrm{GPa}$ for shock loading of single crystal $\mathrm{Cu}$ along $\langle 100\rangle$ at $u_{\mathrm{p}}$ $=0.625 \mathrm{~km} \mathrm{~s}^{-1}$, and it reduces to $13.5 \mathrm{GPa}$ in the bicrystal. Compression plasticity in single crystal $\mathrm{Cu}$ occurs at $u_{\mathrm{p}}$ $\gtrsim 0.75 \mathrm{~km} \mathrm{~s}^{-1}$ (Ref. 11) but it occurs in the bicrystal at lower $u_{\mathrm{p}}\left(0.375 \mathrm{~km} \mathrm{~s}^{-1}\right)$. Thus, GBs may have pronounced effect both on flow strength and spall strength, and void nucleation favors GBs. ${ }^{10}$ However, the regions with maximum tensile stress are not necessary the spall zones, as in these two spallation cases (see below). The tensile strain rate estimated in situ from the particle velocity profile is about $10^{9}-10^{10} \mathrm{~s}^{-1}$

In the $1 \mathrm{D}$ analyses presented above, the structure variation normal to the shock direction is neglected, despite that there exist strong elastic and plastic anisotropy for [00 $\overline{1}]$ and [22ī] loading. Neglecting such spatial variations may lead to incorrect interpretation of the 1D analysis results as regards plasticity and spall, or pose difficulty in the interpretation. $2 \mathrm{D}$ or $3 \mathrm{D}$ analysis is necessary in order for us to gain insight into the microstructure effect on the shock response. Since the initial structure along the tilt or $z$-axis is homogeneous, so the spatial variations in some physical properties along 


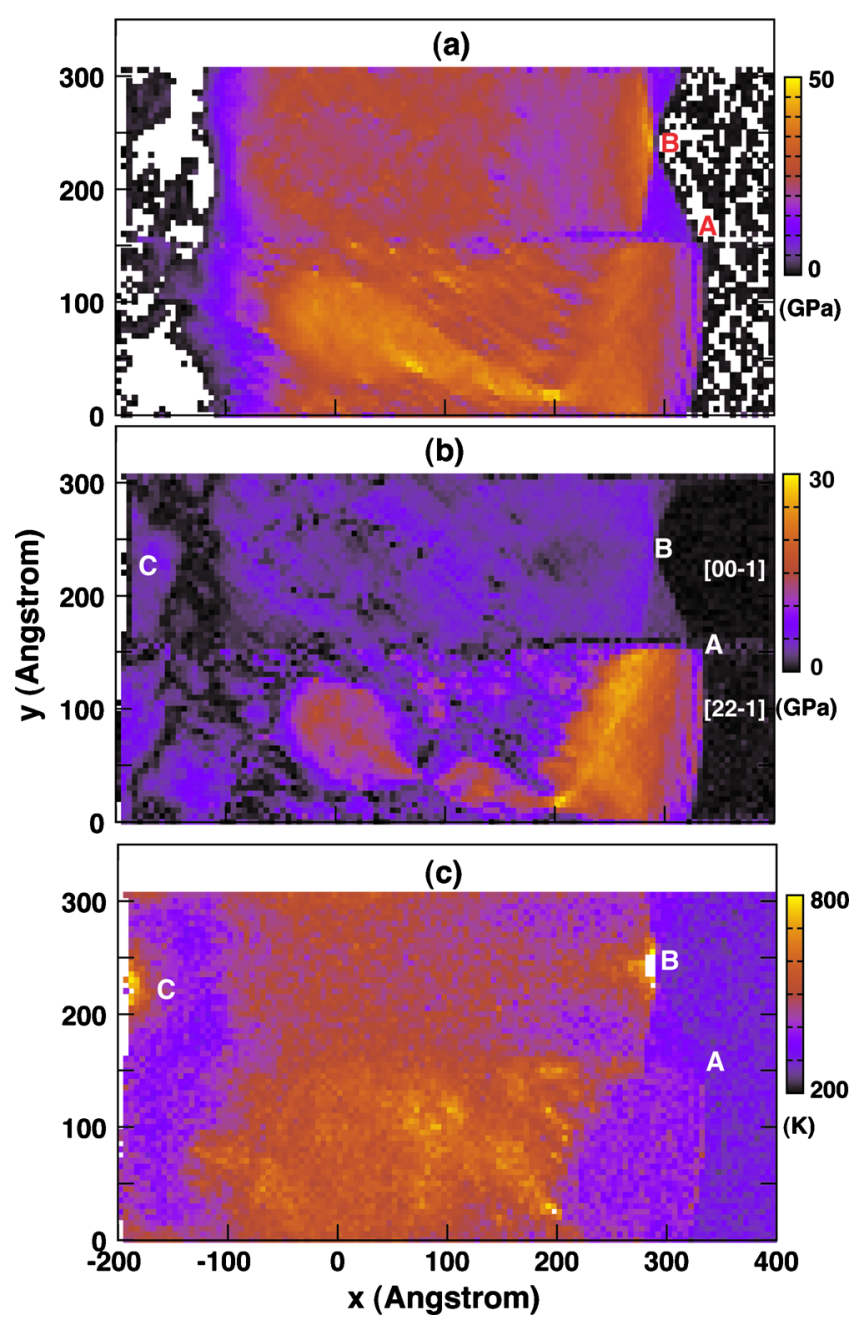

FIG. 5. (Color online) 2D analyses of stress and temperature profiles, for $u_{\mathrm{p}}=0.625 \mathrm{~km} \mathrm{~s}^{-1}$ at $t=6$ ps. (a) $\sigma_{11}(x, y)$, (b) $|2 \tau|(x, y)$, and (c) $T(x, y)$.

this direction is expected to be small, and 2D analysis is largely sufficient (i.e., averaging over the $z$-direction). Figures 1, 2, and 5-10 present the results from the 2D and 3D analyses in terms of local stress, temperature, shear, displacement, and structure.

When subjected to shock compression, plasticity increases with increasing $u_{\mathrm{p}}$, and manifests 2D/3D spatial variations (Fig. 1). Stacking faults (as well as twins; Fig. 2) originating from the GBs are observed for all three cases, since the elevated free energy of GBs reduces the energy

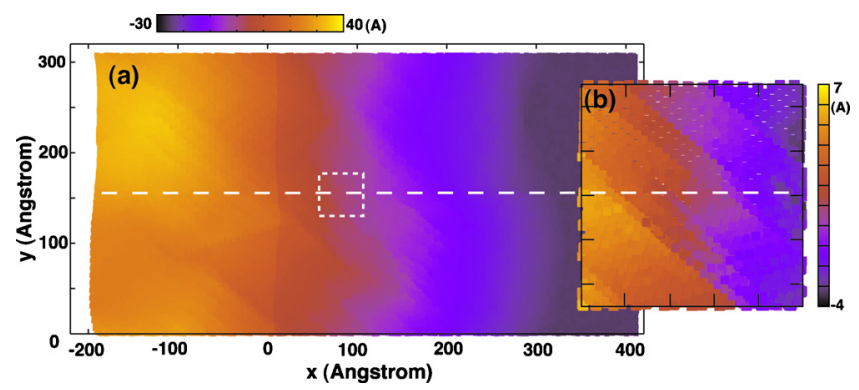

FIG. 6. (Color online) (a) A 2D projection of the displacement field, $D_{1}(x, y)$, for $u_{\mathrm{p}}=0.625 \mathrm{~km} \mathrm{~s}^{-1}$ at $t=6 \mathrm{ps}$. Also see Fig. 1(c). (b) corresponds to the region enclosed by the $50 \AA \times 50 \AA$ square in (a). The longdashed line denotes a GB.

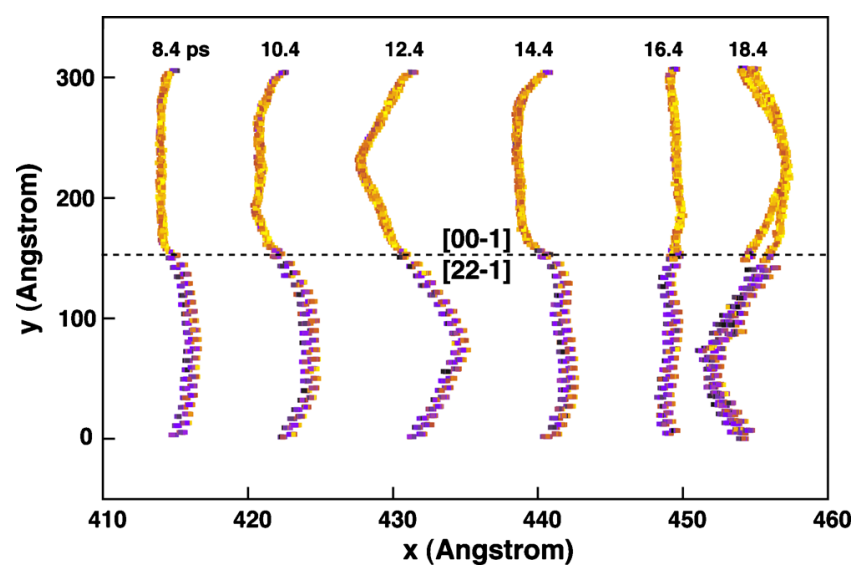

FIG. 7. (Color online) Dynamics of the target free surface after the shock breakout, projected along [1 $\overline{1} 0]$. Color-coding is based on potential energy, and only the atoms near the free surface (with high potential energy) are plotted. $u_{\mathrm{p}}=0.375 \mathrm{~km} \mathrm{~s}^{-1}$.

barrier to plasticity nucleation, while GB free volume and local stress concentration may also play a role. ${ }^{25}$ The number of nucleation sites and shear planes, and the degree of shear deformation increase with increasing shock strength. For $u_{\mathrm{p}}$ $=0.375 \mathrm{~km} \mathrm{~s}^{-1}$, the plasticity is negligible, and GB-initiated dislocations are largely localized. The "elastic precursor" in $u_{\mathrm{fs}}(t)$ is simply an artifact of adding two sets of elastic waves traveling at different velocities. Activation of different variants of $\{111\}$ slip planes (intersecting high-shear planes, Fig. 1) occurs at higher $u_{\mathrm{p}}$. Plastic deformation varies spatially within a grain and differs considerably between grains 1 and 2 ; shear deformation $\left(\eta^{\mathrm{vM}}\right)$ in grain 2 is higher than those in grain 1 and GB regions. The elastic and plastic regions can be identified for $u_{\mathrm{p}}=0.5$ and $0.625 \mathrm{~km} \mathrm{~s}^{-1}$ while their interfaces are not well defined.

Shock compression also induces strong spatial variations

(a)

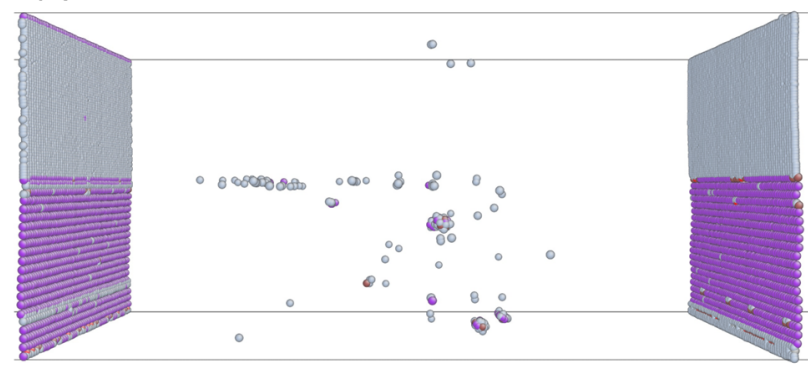

(b)

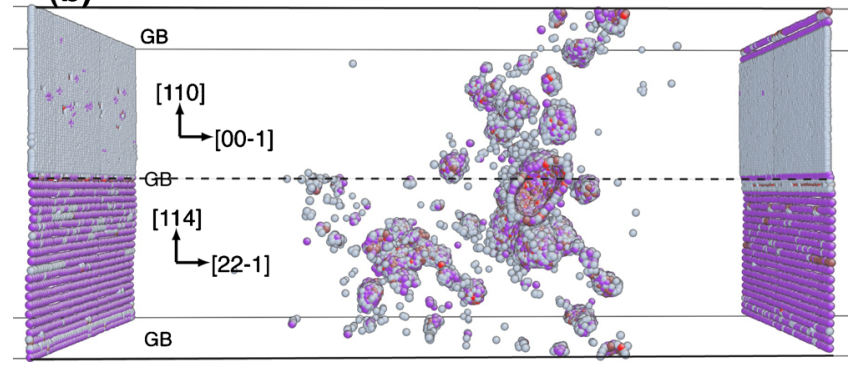

FIG. 8. (Color online) Snapshots of void nucleation and growth at $t$ $=24 \mathrm{ps}$ for $u_{\mathrm{p}}=0.375 \mathrm{~km} \mathrm{~s}^{-1}(\mathrm{a})$, and at $t=23.6 \mathrm{ps}$ for $u_{\mathrm{p}}=0.5 \mathrm{~km} \mathrm{~s}^{-1}$ (b). Only surface atoms are plotted. 


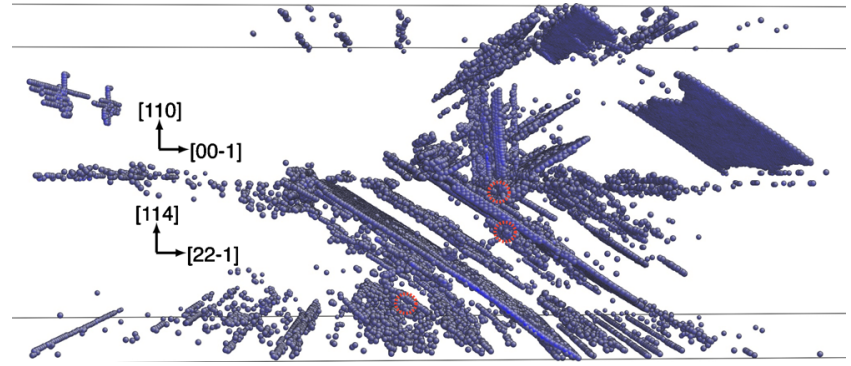

FIG. 9. (Color online) A snapshot of highly shear-deformed regions at $t$ $=18 \mathrm{ps}$ for $u_{\mathrm{p}}=0.5 \mathrm{~km} \mathrm{~s}^{-1}$. Only atoms with $\eta^{\mathrm{vM}} \geq 0.3$ are shown. Some void nucleation sites are indicated with circles.

within grains and across GBs in stress, temperature and displacement profiles (Figs. 5-7). For grain 2 under shock loading with $u_{\mathrm{p}}=0.625 \mathrm{~km} \mathrm{~s}^{-1},|2 \tau|$ peaks in the wave front region $(x \approx 200-330 \AA)$, followed by stress relaxations in the region of $x \approx-50-200 \AA$ with spotted stress concentrations [Fig. 5(b)]. Comparison with the deformation profile [Fig. $1(c)$ indicates that the former represents roughly the elastic region, and the latter, the plastic region. The shear stresses are higher at the spots with less plasticity in the bulk plastic region. Such general and local features in the elastic and plastic deformation are also revealed in $\sigma_{11}(x, y)$ [Fig. 5(a)] and $T(x, y)$ [Fig. 5(c)]. For instance, the temperature is substantially lower in the elastic region than the plastic region. In sharp contrast, it is more difficult to reveal the elasticplastic transition in grain 1 from such profiles. [The elastic region is located within $x \sim 150-280 \AA$ if we also consider the deformation profile, Fig. 1(c).] For grain-1 center, its elastic wave front lags by about $50 \AA$ behind the grain- 2 counterpart. Such observations can be readily attributed to anisotropy in both elasticity and plasticity in the bicrystal ([001] versus $[22 \overline{1}])$. In particular, it is easier to activate the $\{111\}$ slip systems for loading along [001] than $[22 \overline{1}]$ (similar to the Schmid factor effect). The higher critical shear stress in the latter case also induces faster dislocation ava- lanche accompanied by pronounced relaxation in shear stress as seen in Fig. 5(b), so the elastic-plastic transition can be better identified in space in grain 2. At GBs, we observe largely stress relaxation with spotted concentrations in particular in $|2 \tau|$ [Figs. 5(a) and 5(b)]. Heating at GBs [Fig. $5(\mathrm{c})]$ is also observed but it is less pronounced compared to the plastic region within grain 2 where $\eta^{\mathrm{vM}}$ is much higher [Fig. 1(c)].

The observed features in 1D stress profiles in Fig. 3(b) can be interpreted in terms of spatial variations in $\sigma_{11}(x, y)$ [Fig. 5(a)]. The precursor (arrow) and the stress peak (circle) in Fig. 3(b) correspond to point $A$ and $B$ in Fig. 5, respectively. While it is indeed elastic, the precursor does not represent the elastic-plastic transition, since the region immediately behind it $(A B)$ is still elastic. The intersections of the leading elastic wave front in grain 2 with two GBs act as moving point sources which emit perturbations into grain 1 from the GBs; as a result, two envelopes of such perturbations are formed and interact with each other (one is shown as $A B$ ); we refer to this as the "focusing effect." Encountering of these two evolving envelopes gives rise to the stress concentration near $B$, which may further interact with the elastic wave front in grain 1. Consequently, local heating is also induced at $B$ [Fig. 5(c)]. The 1D profile $\sigma_{11}(x)$ peaks at $B$ [Fig. 3(b)] since the stress concentration at $B$ is unsupported in grain 1 , and the plasticity in grain 2 also relaxes the stress.

The nonplanarity of loading induced by the GBs and the anisotropy in elasticity and plasticity (Figs. 1 and 5) can be characterized in terms of displacements as well. For planar loading, $D_{1}$ should be the same for a given $x$, regardless of the $y$-position; the $y$-dependence reflects the extent of deviation from planar loading. Fig. 6 shows strong variation in $D_{1}$ along $x$ and $y$ for shock fronts, "elastic-plastic" transition region and "plastic region," consistent with Figs. 1(c) and 5.

Since only the measurements of surface movement (displacement or velocity) are normally available in experi-

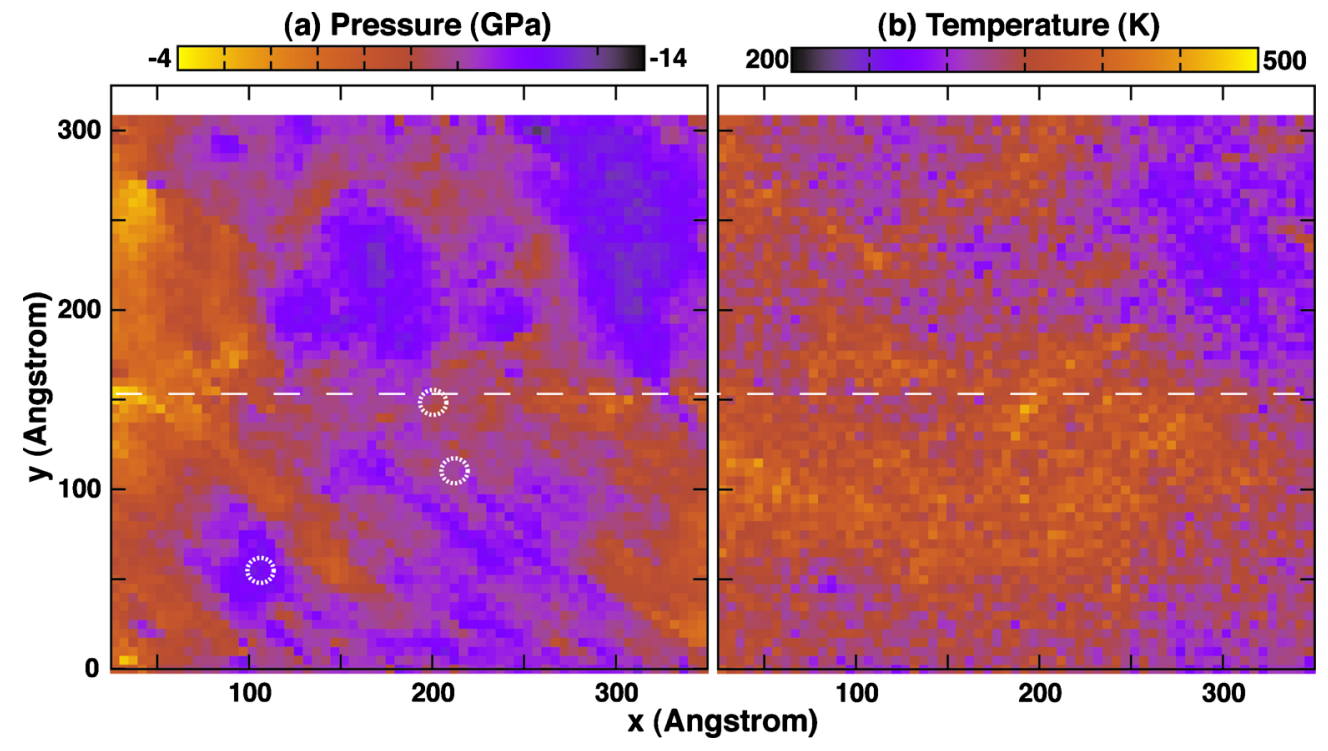

FIG. 10. (Color online) 2D profiles of hydrostatic pressure (a) and temperature (b) at $t=18 \mathrm{ps}$ for $u_{\mathrm{p}}=0.5 \mathrm{~km} \mathrm{~s}^{-1}$. Dashed line: GB; circled areas: void nucleation sites. Also see Fig. 9. 
ments, we also examine the surface movement of the bicrystal after the shock breakout, including surface roughening. ${ }^{13}$ As an example, Fig. 7 shows the position (essentially $D_{1}$ ) of the free surface atoms. Shock breakout first occurs in grain 2 $(t \sim 8.4 \mathrm{ps})$ and then in grain 1; the free surface is distorted as time evolves (roughening). The leading wave front becomes concave rightward for grain 2 and leftward for grain 1 , since the former travel faster but is slowed down at the GBs, and the opposite occurs for grain 1. This dragging effect manifested on the surface is similar to the focusing effect within the interior, and they have the same cause: the crosstalk through GBs to accommodate the elastic (and plastic) anisotropy. The maximum differential displacement is about $7 \AA$ ( $\sim 12.4$ ps $)$. At later times, grain- 1 free surface leads its grain-2 counterpart (18.4 ps) because of the releaseinduced deceleration of grain-2 surface, which also occurs earlier for grain 2 than grain 1. And the surface morphology also changes accordingly.

The difference in wave speeds in grains 1 and 2 (it is $\sim 16 \%$ slower in grain 1), and subsequently, particle velocity across the GBs, may lead to differential displacements across the GBs (Fig. 6) via the focusing effect and dragging effect, and thus friction. In current simulations, friction (including frictional heating) is not a pronounced feature [e.g., Fig. 6(b)], likely because we do not preapply a stress normal to GBs and the GB roughness is minor ${ }^{26}$ (or because of the particular GB characteristics). In addition, the shear strain concentration occurs near the flyer plate free surface $[C$ in Fig. 1(c) during release; the corresponding plastic heating is also observed in Fig. 5(c)], and it may also be due to the focusing effect. MD simulations of the focusing and dragging effects serve as a basis for hydrodynamic or elasticplastic modeling at higher levels. 7,27

The shocks are reflected at the free surfaces as release fans, which unload the shocked materials to zero stresses (and lower temperature) and further into tension and spallation. The structure, shear deformation, stress, and temperature are characterized as regards void nucleation and growth for shock loading with three different $u_{\mathrm{p}}$ (Figs. 8-10). The shear deformation induced by shock compression is not necessarily stable upon release (partially or completely reversed) due to, e.g., the lack of effective dislocation-pinning mechanisms, for the grain size and loading conditions explored here. Under sufficient tensile loading, plasticity (re)nucleates at GBs or grows from the remnant plasticity or defects, and progresses with increasing tensile load in the early stage of void nucleation and growth (shear deformation around a void may diminish as the void growth unloads tension at later stages). Besides well defined stacking faults (as well as twins), local disordering is also observed. The slip "bands" within the grain interiors (Fig. 9) largely correspond to the fcc-hcp structure changes. For $u_{\mathrm{p}}=0.375 \mathrm{~km} \mathrm{~s}^{-1}$, the compression plasticity is negligible while the tensile plasticity is pronounced (in particular for grain 2). Tensile plasticity increases with increasing $u_{\mathrm{p}}$ as well. For $u_{\mathrm{p}}=0.5$ and $0.625 \mathrm{~km} \mathrm{~s}^{-1}$, intersecting $\{111\}$ slip planes are observed during tension. The intersections of such planes normally undergo more disordering and shear. Grain 2 undergoes more plasticity than grain 1 during tension (comparing the area and magnitude of shear) in all cases.

Plasticity, defects, solid state disordering, and their interactions induce local weakening, and consequently, nanovoids nucleate at GBs and the weakened regions of grain interiors. We characterize voids with coordination number $(\mathrm{CN})$ since the surface atoms enclosing a void are undercoordinated. ${ }^{28}$ Nanovoids are revealed by such atoms with $\mathrm{CN} \leq 8$ in selected configurations under tension (Fig. 8). Nanovoids can be of different size and shape during void growth and coalescence. For instance, the sizes of isolated voids range from subnanometer to $5 \mathrm{~nm}$ in diameter (Fig. 8). For $u_{\mathrm{p}}$ $=0.375 \mathrm{~km} \mathrm{~s}^{-1}$, only few isolated sun-nm voids are observed without marked growth. This case is likely incipient spall. At higher $u_{\mathrm{p}}$, the number of voids and their sizes increase considerably, and spallation transitions to intermediate $\left(0.5 \mathrm{~km} \mathrm{~s}^{-1}\right)$ and full spall $\left(0.625 \mathrm{~km} \mathrm{~s}^{-1}\right)$. The damage is more pronounced in grain 2 than grain 1, consistent with their tensile plasticity. The increasing damage with increasing $u_{\mathrm{p}}$ (incipient to full spall) is also in accord with the $1 \mathrm{D}$ analysis of $u_{\mathrm{fs}}(t)$; the spall pullback feature becomes more evident with increasing $u_{\mathrm{p}}$ [Fig. 3(a)].

Although nanovoids are observed within the grain interior as shown above, their nucleation should still be regarded as intergranular nucleation. Figure 9 shows shear deformation in terms of $\eta^{\mathrm{vM}}$; three representative voids (circles), located on and off the GBs, are nucleated from highly sheardeformed sites such as slip planes (related to, e.g., stacking faults). In the configuration at a later time [Fig. 8(b)], the voids are located predominantly on $\{111\}$ slip planes. Thus, the off-GB voids are nucleated on the slip planes or disordered sites ${ }^{29,30}$ that can be traced back to the GBs, and such nucleation is a GB effect. We have shown that there is no "homogeneous" void nucleation for both crystalline and amorphous metallic solids, ${ }^{20,29,31}$ i.e., void nucleation has to start from "defective," "disordered," or highly sheared sites. So void nucleation is essentially a matter of defect or plasticity nucleation. In this regard, void nucleation on slip planes (or defective sites) initiated from GBs is intergranluar in nature. For sufficiently large grain sizes ${ }^{32}$ and appropriate loading, plasticity and thus void may nucleate within grain interiors independent of the GBs (strictly defined intragranular void nucleation). The widely used terminology, intergranular versus transgranular damage, in our opinion, does not differentiate the exact nature of void nucleation and may be better termed as intergranular versus intragranular void nucleation for ductile damage.

The stress and temperature conditions associated with void nucleation are illustrated in Fig. 10, using $u_{\mathrm{p}}$ $=0.5 \mathrm{~km} \mathrm{~s}^{-1}$ as an example. The tensile stress distribution (here pressure rather than $\sigma_{11}$ is used) shows marked spatial variations; the tensile stress concentration sites are not necessarily the void nucleation sites [Fig. 10(a)]. On the other hand, void nucleation occurs at hotter regions [Fig. 10(b)], which correlate well with the shear deformation (Fig. 9), since both elevated temperature and plasticity facilitate void nucleation as shown before. ${ }^{11}$ The weakening effect of GB and GB-initiated plasticity likely plays the key role in void 
nucleation. In some regions with the highest tensile stress, plasticity can be much less pronounced so these regions are effective stronger than some other regions with smaller tensile stresses. This explains why spall does not necessarily occur at regions with the highest tensile stress as seen from $1 \mathrm{D}$ analysis of $\sigma_{11}(x)$ (Fig. 4).

Plasticity and spallation in $\mathrm{Cu}$ bicrystal are intertwined processes during void nucleation and growth, and depend on strain rate and grain characteristics (e.g., grain size, GB energy, and orientations). Of particular interest are the crossovers in time and size scales for intergranular and intragranular void nucleation. As a first attempt on shock response of anisotropic bicrystals (plasticity, spall, friction, surface damage, and roughening), our results show the potential to make connections to spatially resolved measurements and phenomenological modeling at meso- and continuum levels. Accumulating the statics of physics-based MD simulations is crucial for phenomenological modeling. First-principles based MD simulations can help establish such physics-based models as the relations between grain characteristics and surface roughening or shock front broadening. Systematic research efforts in the future are desired along these lines.

\section{CONCLUSIONS}

We have characterized the shock response of $\mathrm{Cu}$ bicrystals with $\Sigma 3(110)_{1} /(114)_{2}\langle 110\rangle$ tilt GBs, including plasticity and spall damage. Our results show pronounced deviation from planar loading due to the GBs and strong anisotropy in elasticity and plasticity, thus invalidating simplified 1D analysis for such anisotropic bicrystals. As a result, strong stress and strain concentrations are also observed. The incipient to full spallation are explored with different impact velocities. Voids nucleate either at GBs or on GB-initiated shear planes, and the spall damage also depends on grain orientation. Such physics-based MD simulations may supply constraints on or serve as a basis for meso- and continuum level modeling of shock response of polycrystalline solids.

\section{ACKNOWLEDGMENTS}

This work is supported by the Advanced Simulation and Computation (ASC) and Laboratory Directed Research and Development (LDRD) programs at LANL. LANL is operated by Los Alamos National Security, LLC for the U.S. Department of Energy under Contract No. DE-AC5206NA25396. We have benefited from discussions with $\mathrm{M}$. Demkowicz, C. Brandl, A. Koskelo, and D. Dennis-Koller.
${ }^{1}$ M. A. Meyers, Dynamic Behavior of Materials (Wiley, New York, 1994). ${ }^{2}$ M. A. Meyers and C. T. Aimone, Prog. Mater. Sci. 28, 1 (1983).

${ }^{3}$ G. I. Kanel, Int. J. Fract. 163, 173 (2010).

${ }^{4}$ G. E. Norman, A. Y. Kuksin, V. V. Stegailov, and A. V. Yanilkin, AIP Conf. Proc. CP955, 329 (2008)

${ }^{5}$ V. Dremov, A. Petrovtsev, P. Sapozhnikov, M. Smirnova, D. L. Preston, and M. A. Zocher, Phys. Rev. B 74, 144110 (2006).

${ }^{6}$ E. M. Bringa, A. Caro, Y. Wang, M. Victoria, J. M. McNaney, B. A. Remington, R. F. Smith, B. R. Torrala, and H. Van Swygenhoven, Science 309, 1838 (2005).

${ }^{7}$ M. Meyers and M. Carvalho, Mater. Sci. Eng. 24, 131 (1976).

${ }^{8}$ F. Cao, I. J. Beyerlein, F. L. Addessio, B. H. Sencer, C. P. Trujillo, E. K. Cerreta, and G. T. Gray III, Acta Mater. 58, 549 (2010).

${ }^{9}$ P. Peralta, S. DiGiacomo, S. Hashemian, S. N. Luo, D. Paisley, R. Dickerson, E. Loomis, D. Byler, and K. J. McClellan, Int. J. Damage Mech. 18, 393413 (2009).

${ }^{10}$ S. N. Luo, T. C. Germann, and D. L. Tonks, J. Appl. Phys. 107, 123507 (2010).

${ }^{11}$ S. N. Luo, Q. An, T. C. Germann, and L. B. Han, J. Appl. Phys. 106, 013502 (2009)

${ }^{12}$ W. M. Trott, L. C. Chhabildas, M. R. Baer, and J. N. Castaneda, AIP Conf. Proc. CP620, 845 (2002).

${ }^{13}$ D. L. Paisley, S. N. Luo, S. R. Greenfield, and A. C. Koskelo, Rev. Sci. Instrum. 79, 023902 (2008)

${ }^{14}$ S. N. Luo, D. C. Swift, T. E. Tierney IV, D. L. Paisley, G. A. Kyrala, R. P. Johnson, A. A. Hauer, O. Tschauner, and P. D. Asimow, High Press. Res. 24, 409 (2004).

${ }^{15}$ M. A. Tschopp and D. L. McDowell, Philos. Mag. 87, 3147 (2007).

${ }^{16}$ J. Li, Model. Simul. Mater. Sci. Eng. 11, 173 (2003).

${ }^{17}$ Y. Mishin, M. J. Mehl, D. A. Papaconstantopoulos, A. F. Voter, and J. D. Kress, Phys. Rev. B 63, 224106 (2001).

${ }^{18}$ J. Stadler, R. Mikulla, and H.-R. Trebin, Int. J. Mod. Phys. C 8, 1131 (1997).

${ }^{19}$ B. L. Holian, Shock Waves 5, 149 (1995)

${ }^{20}$ S. G. Srinivasan, M. I. Baskes, and G. J. Wagner, J. Appl. Phys. 101, 043504 (2007)

${ }^{21}$ D. C. Rapaport, The Art of Molecular Dynamics Simulation (Cambridge University Press, Cambridge, 1995).

${ }^{22}$ S. N. Luo, T. C. Germann, and D. L. Tonks, J. Appl. Phys. 107, 056102 (2010).

${ }^{23}$ F. Shimizu, S. Ogata, and J. Li, Mater. Trans. 48, 2923 (2007).

${ }^{24}$ C. L. Kelchner, S. J. Plimpton, and J. C. Hamilton, Phys. Rev. B 58, 11085 (1998).

${ }^{25}$ K. S. Kumar, H. Van Swygenhoven, and S. Suresh, Acta Mater. 51, 5743 (2003).

${ }^{26}$ J. E. Hammerberg, B. L. Holian, T. C. Germann, and R. Ravelo, Metall. Mater. Trans. A 35, 2741 (2004).

${ }^{27}$ J. N. Johnson, J. Phys. Chem. Solids 35, 609 (1974).

${ }^{28}$ S. N. Luo, L. Q. Zheng, Q. An, and S. J. Zhao, Int. J. Mod. Phys. C 17, 1551 (2006).

${ }^{29}$ S. N. Luo, T. C. Germann, and D. L. Tonks, J. Appl. Phys. 106, 123518 (2009).

${ }^{30}$ Y. Ashkenazy and R. S. Averback, Appl. Phys. Lett. 86, 051907 (2005).

${ }^{31}$ B. Arman, S.-N. Luo, T. C. Germann, and T. Çağın, Phys. Rev. B 81, 144201 (2010).

${ }^{32}$ We have performed MD shock simulations of symmetric $\Sigma 11 \mathrm{Cu}$ bicrystals and found that intragranular void nucleation occurs at grain sizes above $\sim 28 \mathrm{~nm}$ (unpublished results). However, this critical size depends on GB nature and loading. 